\title{
Spinal Cord Injury: Present and Future Therapeutic Devices and Prostheses
}

\author{
Simon F. Giszter \\ Neurobiology and Anatomy, Drexel University College of Medicine, and School of Bioengineering and Health Sciences, Drexel
} University, Philadelphia, Pennsylvania 19129

\begin{abstract}
Summary: A range of passive and active devices are under development or are already in clinical use to partially restore function after spinal cord injury (SCI). Prosthetic devices to promote host tissue regeneration and plasticity and reconnection are under development, comprising bioengineered bridging materials free of cells. Alternatively, artificial electrical stimulation and robotic bridges may be used, which is our focus here. A range of neuroprostheses interfacing either with CNS or peripheral nervous system both above and below the lesion are under investigation and are at different stages of development or translation to the clinic. In addition, there are orthotic and robotic devices which are being developed and tested in the laboratory and clinic that can provide mechanical assistance, training or substitution after SCI. The range of different approaches used draw on many different aspects of our current
\end{abstract}

but limited understanding of neural regeneration and plasticity, and spinal cord function and interactions with the cortex. The best therapeutic practice will ultimately likely depend on combinations of these approaches and technologies and on balancing the combined effects of these on the biological mechanisms and their interactions after injury. An increased understanding of plasticity of brain and spinal cord, and of the behavior of innate modular mechanisms in intact and injured systems, will likely assist in future developments. We review the range of device designs under development and in use, the basic understanding of spinal cord organization and plasticity, the problems and design issues in device interactions with the nervous system, and the possible benefits of active motor devices. Key Words: Spinal cord, neuroprostheses, plasticity, rehabilitation, motor function.

\section{INTRODUCTION}

Spinal cord injury (SCI) incurs significant economic costs and has a range of significant impacts on quality of life and longevity. Presently, therapies are limited for the approximately 2.5 million sufferers worldwide, and the possibilities brought into the public eye and hopes for prospective treatments as highlighted by Christopher Reeve and other sufferers since then remain distant. However, steady but limited incremental progress is occurring in several areas. ${ }^{1-6}$ Our goal here is to review this progress (particularly, although not exclusively, as applied to motor prostheses), and to describe the resources to further explore the different aspects of this very broad area. To this end, we will briefly describe some current systems (specifically the Freehand system [NeuroControl Corp., North Ridgeville, $\mathrm{OH}]$ as a case

Address correspondence and reprint requests to: Simon F. Giszter, $\mathrm{Ph} . D .$, Neurobiology and Anatomy, Drexel University College of Medicine, 2900 Queen Lane, Philadelphia, PA 19129. or E-mail: sg33@drexel.edu. study), we will provide an overview of the issues arising in designing present and future motor prostheses from a motor control standpoint, and we will survey the current available technologies and their possible extensions and future applications.

Given the range of segmental levels and severity of injury that can occur, there are a variety of needs and issues to be explored. For example, surveys of paraplegic patients indicate that leg use is significantly less of a priority than bowel, bladder, sexual function and autonomic dysreflexia. ${ }^{7}$ For quadriplegic patients, arm and hand manipulative skills take priority. Freedom from ventilators is primary for high quadriplegia. Pain and spasticity are issues for most individuals with spinal cord injuries across the range of the segmental levels and degrees of completeness of injury. Several of the nonmotor issues arising are addressed in detail in other articles in this volume. We will primarily focus here on restoration of motor control and its associated technologies. However, some of these techniques and therapies may have applications broader than motor control. 
SCI therapies can be divided into several classes after the immediate emergency treatment and stabilization after trauma. Some treatments target the immediate changes after the injury, minimizing inflammatory and apoptotic reactions. Other approaches seek to restore or substitute lost functions by means of cellular transplants of various types: 1) autologous or heterologous peripheral nerve, Schwann cells, olfactory cells, 2) embryonic CNS (spinal or other) tissues or stem cells and progenitors, 3) adult stem or progenitor cells, 4) geneticallyengineered cell lines, and 5) activated macrophages. Yet other approaches use a range of noncellular molecular therapies to attempt to control the responses and plasticity of injured tissues over the long term. For example, agents causing alterations to the extracellular matrix (such as chondroitinase ${ }^{8}$ ) and artificial (or prosthetic) tissue scaffolds and substitutes ${ }^{2}$ may be used to promote plasticity and possible regeneration of the injured tissues. ${ }^{9}$ In general, these approaches are reliant on the "good sense" of the injured CNS to find appropriate cellular targets in sprouting, regeneration or novel circuitry to promote improved function. ${ }^{1,2}$ However, in principle, plasticity can be both an ally and an enemy in the sequelae of the SCI and recovery of function. ${ }^{10,11}$ Many developmental "signposts" are transient, whereas critical periods and other activity-dependent cues may not be readily recapitulated in the injured spinal cord. With these issues arising, surgical interventions organizing completely novel but more predictable connectivity have been proposed, developed and tested in animal models. Such interventions may finesse some of the difficulties arising in re-establishing a functional circuitry. ${ }^{12}$ Finally, active devices may be used to bridge, activate, train, augment and substitute various motor control functions compromised in the injured spinal cord and CNS. These latter devices are the main target of this review and will be elaborated on in more detail as follows.

The likelihood is that ultimately, combinations of cellular therapies, acellular therapies and passive and active prostheses may be needed for the best outcomes after SCI. Combinations present their own set of problems: a combinatorial explosion occurs leading to increasing complexity in an animal study or clinical trial design with increasing needs for more and more subjects to obtain statistical significance. ${ }^{13}$ However, despite these daunting considerations, it is likely to be useful to always keep in mind the differing approaches and possible synergies, especially in considering novel devices and designs.

\section{CURRENT DEVICES AND PROSTHETICS}

From the point of view of the Food and Drug Administration (FDA), devices exclude cellular therapies. Active devices and passive, bioengineered matrices for repair form FDA defined neuroprostheses. ${ }^{14,15}$

\section{Prosthetic device classification}

In considering prostheses in SCI we can examine them from several perspectives: by target function and role, by target tissues and interface or by technical type.

Passive spinal cord prostheses or devices based on artificial matrices combined with FDA-approved neurotrophins and cytokines to promote cell survival, regeneration and plasticity for novel connections are currently at the stage of experimental animal testing. Based on target function, active spinal cord prostheses are currently available for: 1) pain management, ${ }^{16}$ 2) bowel bladder management, ${ }^{17} 3$ ) spasticity management ${ }^{18}$ and 4) motor control of limbs and trunk. These are detailed in the following sections.

In addition there may be a place for active spinal prostheses in autonomic dysreflexia. Autonomic dysreflexia is a little explored area from the perspective of prostheses, but several clinical reports suggest that it may be an interesting target for devices, and autonomic control devices are under development. ${ }^{19,20}$ Dysreflexia can be an indicator of other issues in patient management, and its suppression might require other monitoring.

In a prosthetic classification based on degrees of invasiveness and target tissues, prosthetics range from temporary prosthetic applications used as rehabilitative robotic tools through externally worn passive or active orthotic interventions in the periphery, peripheral transcutaneous stimulators, implanted peripheral functional electrical stimulation designs, dorsal root ganglion and root implanted multi-electrode implants, epidural and intraspinal stimulators and cortical brain machine interfaces. $^{4,5,21}$

Each level of intervention carries with it a differing set of issues, both from basic science and clinical perspectives. Clearly, the ideal intervention is a "smart" prosthetic that is minimally invasive with maximal bandwidth for recording, stimulating or functional capability. In practice, we are limited by our current technology, by our understanding of basic science of neural functions and by our understanding of user interface needs in a prosthetic device. These limits force compromise approaches that can seem relatively "dumbed down." However, there is increasing pressure and impetus to move beyond these limits in technology and understanding. This impetus is coming jointly from public, government and academic communities. ${ }^{22-24}$ Much of this focus of attention is on the difficult issues of motor function restoration.

\section{Clinical devices for the motor system after SCI}

Probably the most successful current applications of motor prostheses in SCI have been in tetraplegia and hemiplegia. The hand-grasp system, with augmentative surgical procedures in the hand, has been very effective and has improved quality of life for hundreds of individ- 


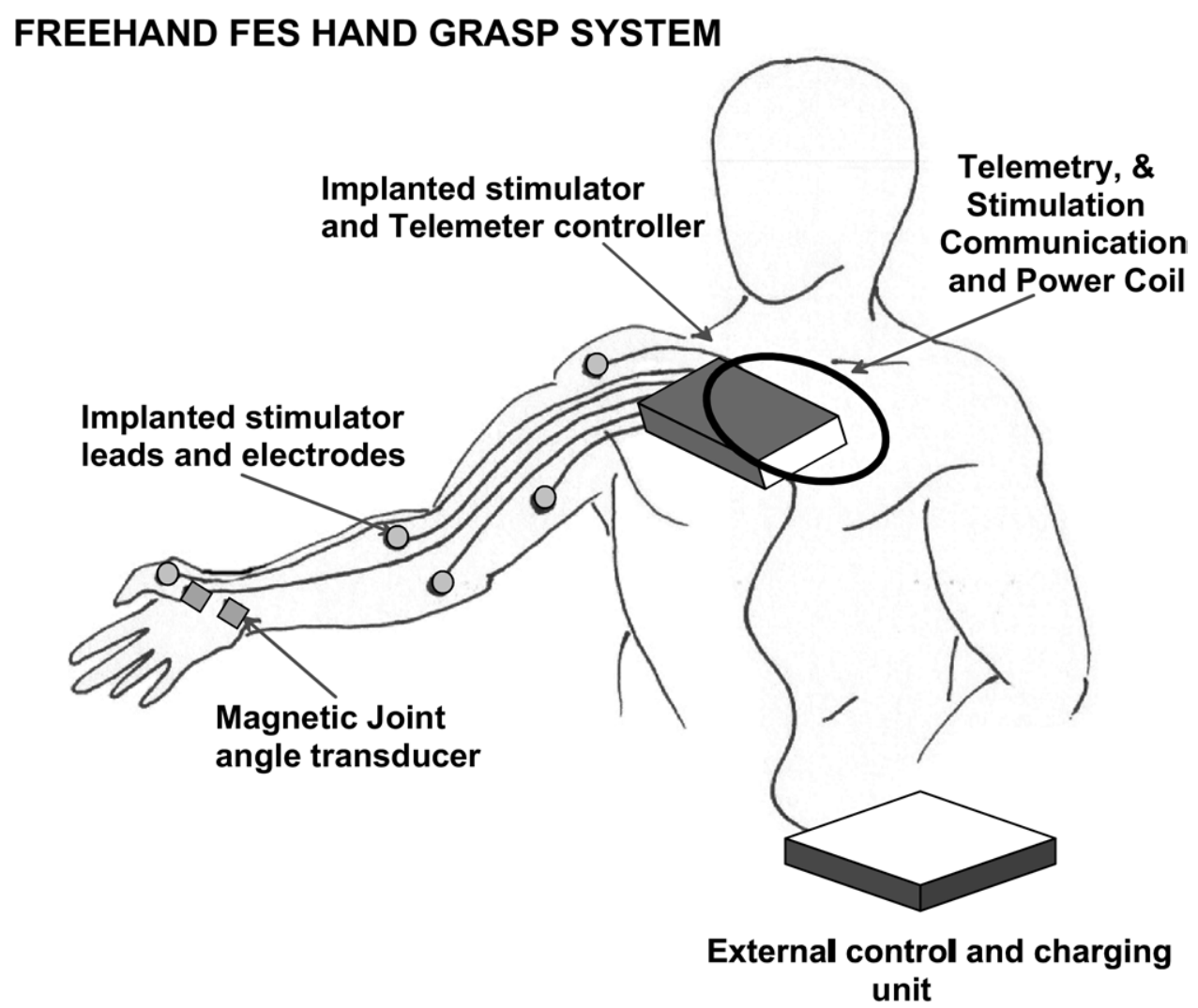

FIG 1. The Freehand Hand Grasp system. The second generation device is fully implantable, excepting the grasp controller. Sensing from the implanted magnetic joint angle transducers is used to detect wrist motion. This is communicated to the external controller through the coil. Programmed stimulation and power of the implanted hardware is also provided through this coil. Epimysial electrodes are implanted for stimulation. Proportional control of grasp is controlled through wrist or shoulder motion. Used for C5/C6 motor complete injuries; the system has a very significant impact on activities of daily living for the recipients.

uals (see as follows). A range of foot drop prostheses have application to hemiplegia. Standing and transfer prostheses in C6 to T9 SCI have also been very effective. These have been very thoroughly reviewed. ${ }^{25-27}$ The technologies available for prostheses are evolving rapidly and a range of novel therapies may also rapidly become more feasible. The control options, designs and strategies, and animal experiments supporting them will form the basis of the remainder of this review.

\section{The Freehand system: a case study}

The Freehand system is very well designed prosthetic system, which has gone through several generations, extensive testing and commercialization. ${ }^{28}$ It demonstrates most, if not all, of the basic issues in providing a device to spinal cord injury patients.

The goal of this prosthesis is to provide a hand grasp to individuals with motor complete injury at the C5-C6 level. Stimulation strategy, control methods and user interface for the device are well worked out (Fig. 1). Epimysial electrodes are implanted in the muscles of the hand and forearm. These muscles are stimulated to generate grasp forces. In the first generation devices, stimulation for grasp was controlled by motions of the shoulder, which were mea- sured by using external goniometry. This strategy is feasible in both C5 and C6 injuries. Stimulation patterns are customized to the individual, there is rehabilitation support and where needed, augmentative surgical procedures and tendon transfers may be added. Thus, a broad and integrative approach to restoration of function is taken by using a team of clinicians and engineers.

In the later iterations, in a refinement for C6 patients, grasp force is instead generated based on wrist motion detected with implanted sensors. Control of the stimulation is derived using coordination with the tenodesis grasp in use by subjects before device incorporation. This wrist-based control is significantly more intuitive than that used in the earlier device designs, which used shoulder-based controls. Pinch forces of up to 25 Newtons can be achieved. The device improves activities of daily living, and patient satisfaction with the device is high. Quality of life was reported to be improved in about $88 \%$ of the users surveyed. The implanted stimulation systems are preferred to surface or percutaneous systems on several grounds. The system was approved by the FDA in 1997, and a company was formed to commercialize the device. Despite these many successes, the device itself is no longer a central component 
of the business strategy of the commercializing company, and significantly fewer new systems have been implanted than were anticipated since FDA approval. The devices have continued to be supported as a pro bono legacy, but the patient base was much smaller than initially estimated, to the point that revenues were reportedly insufficient to support a major focus of company operations. This issue arises particularly as more lucrative opportunities and revenue streams occur based on company intellectual property and expertise. The clinical applications are then effectively "orphaned." This outcome is a significant concern for the developer and commercializer of the clinical prosthetic technology. This is a real potential pitfall in ensuring that needed devices reach the patient population and continue to be improved after their initial commercialization.

\section{Therapeutic benefits of spinal cord prostheses}

Benefits of bladder and pain prostheses, the BION systems (under development and clinical testing through the Alfred E. Mann Institute, University of Southern California), and brain computer interfaces (BCIs) are documented in other articles in this volume. There are a range of more or less established benefits of spinal cord motor prostheses that have been documented for clinically implemented systems ${ }^{29-34}$ as reviewed by Barbeau and colleagues ${ }^{29}$ and by Dobkin. ${ }^{30}$ Functional electrical stimulation (FES)-assisted walking systems with peroneal nerve stimulation and locomotor training provided both faster and more efficient (lower oxygen consumption) locomotion. H-reflex testing showed that reflex modulations had weakened after SCI were improved. Ankle stiffness measures were altered, with intrinsic stiffness gain increasing by $97 \%$, passive stiffness gain decreasing by $39 \%$ and reflex stiffness gain decreasing by $89 \%$. Prostheses can be used to improve range of activities of daily living, effect physiological changes and ambulatory systems in the future may remove accessibility barriers that remain in many kinds of travel or activity, despite increased removal of environmental barriers for wheelchair use in many cultures. The likelihood is that a range of other issues, such as pressure sore incidence, pain levels, hyperreflexia and autonomic dysreflexia may be influenced positively by motor training or prostheses. In animal models, a range of benefits of activity in cardiovascular function, muscle mass, bone density and presence of trophic factors in the CNS and periphery have been examined rigorously. ${ }^{35-38}$

\section{CONTROL AND DESIGN STRATEGIES FOR CURRENT AND FUTURE MOTOR PROSTHESES: A MOTOR CONTROL PERSPECTIVE}

The ideal motor prostheses are easy to use and adaptable to many tasks. The goal of this section is to provide an overview of how the design of prosthetics may be impacted by our current understanding of motor organization and motor learning. In considering how to achieve such a smart prosthetic for use after SCI it makes sense to consider the operation of the intact spinal cord and its contributions to motor control.

\section{The intact system}

Normal motor function and the role of the spinal cord are both important in current and future prosthetic designs. A key issue in normal motor control and its development that may act as a major constraint on the organization of movement and the extent of hierarchy in its control and development is the "degrees of freedom" problem. This is also an issue for the designer of a prosthetic and ideally the engineered and biological solutions blend seamlessly.

The motor control problem and the degrees of freedom problem. Consider a motor task such as intercepting and trapping a soccer ball with the foot. The pass is a poor one and the ball is traveling about 3 feet above the ground. The nervous system must accomplish several things. The ball trajectory must be rapidly predicted, the range of possibilities on the field evaluated, a plan of action selected, a kinetic plan elaborated and this plan implemented and executed adaptively based on conditions encountered. The implementation and execution stage alone is of daunting complexity. An excess of degrees of freedom is available at the joint level for positioning the limb. There is also a significant redundancy in the muscles available to generate torques at these joints, and in choosing the compliances of the joints through the motion. This embarrassment of riches make the issue of choosing the best limb postures, the best trajectories and the best muscle activation patterns potentially very complex (the so-called "curse of dimensionality"). For example, Wolpert and Ghahramani ${ }^{39}$ point out that human "muscle space" comprises about 600 muscles. Considering only binary activation of these muscles leads to $2^{600}$ patterns, which equals a number larger than the estimated count of atoms in the known universe. The first clear statement of the degrees of freedom problem in motor control outlined here was made by Bernstein. The available degrees of freedom are powerful, if engaged appropriately. They enable immense flexibility and afford rapid use of novel tools and new motor strategies. Similar forms of leg motions to those intercepting a pass might instead be adapted to kick the soccer ball into the goal, to execute the grand battement of a dancer or to drive a crescent kick designed to break bone by a martial artist. Each has a different goal in terms of the kinematics, the kinetics, and the control of force and interaction with external objects; therefore, different muscular and joint compliances are needed. All of these are learned. Stepping over ground has much in 
common with these motions, but it also seems to have a much stronger innate component. The process of learning may be "bootstrapped" by such innate mechanisms.

Spinal capabilities. Historically, strong evolutionary pressures have favored low level neural structures that support a basic repertoire of movements. Consider a wildebeest calf born on the plains of Africa or a hatchling turtle on a Caribbean beach. Both will soon be devoured if they fail to rapidly organize directed locomotion. In fact wildebeest calves move with the herd within a few hours, and the mechanisms supporting this movement have significant spinal cord interneuronal circuitry components.

Spinal circuitry: pattern generation and modularity. The basis for the rapid organization of locomotor function in the wildebeest seems to be a set of modular spinal cord structures. These comprise modular pattern generators and, more controversially, primitives. ${ }^{40}$ These represent an innate capacity to organize behaviors like locomotor stepping. These capacities were preserved through evolution and clearly persist into the human infant. ${ }^{41}$ Modular organization can be extracted statistically and examined in intact adult human locomotion and reaching. ${ }^{42}$ Central pattern generators represent the capacity to generate basic patterns such as locomotor gait, breathing or swimming in the absence of any phasic sensory feedback in isolated CNS, such as lumbar spinal cord. ${ }^{43}$ Primitives represent modular subunits of these patterns that are re-used and may be recruited in flexible ways. This strategy helps the CNS to rapidly adapt and correct the innately structured patterns across different context and perturbations. ${ }^{44}$ It is believed that both pattern generators and primitives may help "bootstrap" skilled movement and remain as components of voluntary movement. Some of the execution details and coordination of voluntary movements may be deferred to these spinal systems, freeing the brain of some execution concerns so that we can talk as we run.

Corticospinal control and augmentation of spinal pattern generation. When the cortex organizes highly practiced voluntary movements, it is likely that it has expectations of the modularity and behavior of these spinal systems. Some of these are built through development, and there is likely to be co-adaptation in the corticospinal system through development. Cortical representations provide the fractionation and flexibility needed to move beyond the automaticity of lower animal motor controls. After postnatal day 1 or day 2, thoracic spinal transection in the compensatory corticospinal adaptations and representations of a rat may play a major role in $20 \%$ of such rats that develop weight-supported autonomous locomotion of hind limbs, despite complete thoracic spinal transection. ${ }^{4,46}$ The corticospinal interface is adapted and elaborated in critical periods. ${ }^{47}$ It is hypothesized that the P1/P2-injured rats are capable of learning cortical (and other) control of "the new interface" generated in thoracic spinal transection, and in ways that adult injured rats are not, after being long past their critical period plasticity.

Brain-driven plasticity in spinal cord. The cortex, in cooperation with other descending pathways, is also now known to be able to drive plastic changes in the spinal cord. Cortical and cerebellar mechanisms are involved in embedding a variety of long-term changes in the spinal cord. ${ }^{3}$ In monkeys, rats and people, the $\mathrm{H}$ reflex can be conditioned to increase or decrease its gain. These changes become embedded in the spinal circuitry. However, such plastic mechanism effects do not abolish pattern generation or spinal reflexes, such as grasp. Instead they are likely to play roles adapting them more or less subtly to novel task demands and styles of motion. In animal models, after peripheral cutaneous nerve lesion in cats, there is significant compensation, restoring locomotor patterns. Much of this compensation becomes embedded in the spinal mechanisms. ${ }^{48}$ However, we do not fully understand how important the continued shaping is by these descending mechanisms. Such overt shaping is clearly lost in severe SCI.

Intrinsic spinal plasticity. The spinal cord circuitry and its pattern generator systems exhibit an intrinsic goal-directed plasticity even in isolation. This has been demonstrated across species in vitro ${ }^{49,50}$ and in vivo. In the instance of the peripheral nerve lesions ${ }^{51}$ explored by Bouyer and Rossignol, ${ }^{48}$ the spinal cord of cats experiencing the peripheral lesion prior to spinal transection fares worse than a cat with neurectomies postlesion in the short term. Presumably, this is a reliance on descending controls and inputs developed before the lesion. However, both types of spinalized cats ultimately show measures of compensation due to intrinsic and purposive plasticity. The spinal cord in isolation exhibits a range of adaptive responses that occur on multiple time scales, balancing contributions of different reflexes based on cutaneous or muscle nerve lesions ${ }^{48,51,52}$ or training and motor experience postlesion. ${ }^{53}$ These training effects are seen in the clinic as well as the laboratory. ${ }^{54}$

The intrinsic plasticity of spinal cord, its modular functionally organized circuitry and its capability to organize (locally) purposive movement is partly the basis of several rehabilitation approaches.

Spinal organization as a set of potential targets.

The principal upshot and take-home message of this discussion is that spinal cord prosthetics may be able to use these modular spinal cord systems. This is possible to the extent that the modular spinal systems persist and remain functional after SCI, and that they can be sensibly recruited and adapted to use. These modular mechanisms automatically solve certain degrees of freedom issues, recruit motor pools in size order and may interact in a sensible way with other spinal circuits. Thus, they may 
considerably simplify parts of the task of construction of prostheses and comprise systems that are more intuitively controlled, whether directly by the brain or less directly through the use of prostheses. In addition, various prostheses organized extrinsic to spinal cord circuitry may very well alter the behavior of the spinal cord systems separated from voluntary control due to their intrinsic plasticity. These interactions could assist or interfere with improved function and the effect will depend on the design, context and training used with the prostheses.

\section{Strategies for restoring functions after SCI}

After SCI, descending control of specific motor pools needed for specific tasks may be lost. The local spinal controls may be intact or damaged, but are altered by nature of the lost descending control. Sprouting by neurons both below and above the lesion to occupy synaptic territories vacated by severed or dying axons may be either assistive or destructive in useful function postlesion. Different strategies for prostheses construction attack these issues in very different ways.

\section{Direct motor pool controls}

By far the most common prosthetic strategy is to identify muscles needed for tasks, but lost to descending controls, and to provide a new channel of conscious control to the user usually by peripheral or surface stimulation. Thus, the user of the Freehand hand grasp system uses shoulder muscle control to activate and regulate appropriate finger muscles that are no longer accessible by descending control to execute functionally appropriate grasp, grip and carrying operations. The strategy need not be "normal" and is an open loop in the sense that the feedback and knowledge of results is purely visual.

\section{PATTERN COMPLETION AND INFERRING MOTOR INTENTS}

An alternate strategy employed by Kirsch and colleagues $^{55}$ is to use a combination of electromyogram (EMG) recordings in intact individuals, biomechanical modeling and modeling of residual capabilities through the FES system to design a "pattern completion" style of control. Based on the muscles voluntarily recruited, the system uses predicting algorithms to pattern match and complete controls of the FES controlled portions of musculature. If motor pool loss has occurred, the biomechanical modeling allows a best match of the residual muscle activation to achieve the needed mechanics. The user thus does not learn odd or novel patterns, but rather the prosthesis uses the muscle activation patterns normally used to infer the appropriate prosthetic control. This is a strategy applicable to FES or exoskeleton and orthotic control. It is also partly the basis of the foot drop prostheses for hemiplegia (e.g., dorsiflexing the foot whenever the patient flexes his or her hip). It relies on the modularity or stereotypy of normal proximal muscle function. In principle, the prosthesis and user might cooperate to expand the functional domain.

\section{STIMULATION TO ACTIVATE RESIDUAL FUNCTIONS}

Stimulation of either peripheral or central structures can be used to activate or augment the residual functions in the spinal cord below the lesion. This technique has been used with peripheral nerve stimulation to drive flexion via spinal reflex circuits. Work on intact and computer-modeled cats and rats is gradually unraveling the range of sensory feedback mechanisms that trigger, regulate and phase reset the gait pattern and stance and swing at segmental levels. ${ }^{56}$ By tapping into these systems, a locomotor "pattern completion" or augmentation may be achieved when nerve or skin stimulation can selectively recruit the different controls. This strategy was introduced early. ${ }^{57}$ It is used in the Parastep system (Sigmedics, Inc., Fairborn, OH) ${ }^{58}$ wherein patients can control their own legs through switches on a walker, and the various foot drop systems. It is being tested more broadly in a cat model of locomotion and gait control, by Rybak and colleagues. ${ }^{59}$ They use fictive circuit analysis of spinal cord, neural and biomechanical modeling, and detailed EMG and kinematic recording to tune their intervention strategy. The notion is that the prosthetic builder will design and tune the system based on principled modeling and data acquisition to achieve gait stability and desired kinetic and kinematic outcomes.

Using H-reflex training in incomplete SCI in a rat model, Wolpaw ${ }^{3}$ and Chen and colleagues ${ }^{60}$ have now demonstrated that device-based training can promote gait compensation. The use of devices in combination with residual plasticity and function to steer plastic changes in appropriate directions is a very exciting prospect.

\section{THE NUTS AND BOLTS OF PROSTHESES FOR RESTORATION OF MOTOR FUNCTIONS AFTER SCI}

Motor prostheses perform several sets of tasks depending on whether they are designed as open-loop or closed-loop devices with the user and environment, and depending on the autonomous computation and control embedded in the prosthetic. The goal of this section is to overview the technical options in prosthetics design.

\section{Prostheses structures}

All perform at least three basis tasks and are used in specific contexts (Fig. 2): 1) acquisition of user signals, 2) processing/control, 3) actions: stimulation/output, and

4) protocol/context/application switching or enabling. 


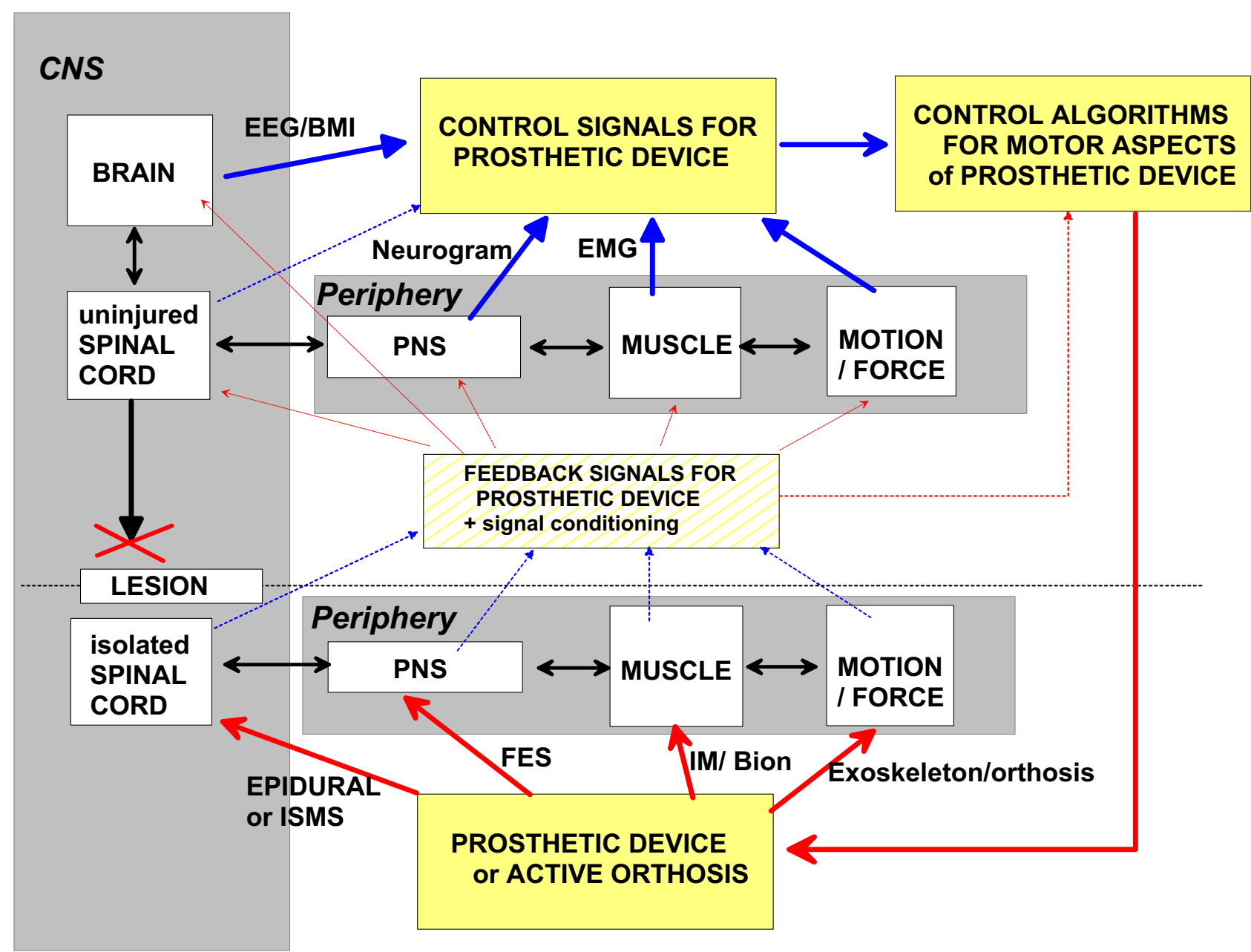

FIG 2. Current and future routes for spinal cord devices: grey background blocks: CNS/periphery. Yellow blocks: prosthetic components. Control signals may be drawn from intact CNS or periphery or user motions (solid blue arrows). These can be used to control orthoses or spinal or peripheral stimulation to augment the residual function (solid red arrows). In principle, prosthetic feedback drawn from CNS or periphery below the lesion could be provided to the user (dotted red and blue) or used in the device. In practice, this is not currently used to a significant extent. The text reviews the various elements and technologies in this scheme in more detail. This diagram indicates the many possible routes that could be used for restoring and providing function. BMI = brain machine interface; EEG = electroencephalogram/electrocorticogram; EMG = electromyogram; IM/Bion = intramuscular, epimysial or BION stimulation; FES = functional electrical stimulation; PNS = peripheral nervous system.

In addition a smarter prosthetic ${ }^{22}$ may incorporate feedback and local controls: 1) "smart prosthetic" acquisition of information about the environmental state, 2) processing/control and integration of user and environment information, 3) delivery of actions at user and/or device environment or target interfaces.

Spinal prostheses will always interact with the nervous system, either directly or indirectly, both above a spinal cord lesion and below the injury (Fig. 2). Above the lesion, user intention and intact voluntary systems are used in some way to drive the prosthetic. There may also be direct feedback through some sensory prosthetics to mimic normal kinds of sensation. Below the lesion, the prosthesis either directly recruits the nervous system or can elicit responses and plasticity in the parts of the spinal cord separated from brain controls through imposed motions on the periphery. These interactions may make design of appropriate controls for a prosthetic quite complicated. Depending on the residual function, severity of injury and type of control provided by the designer of a prosthetic, the brain may be asked to take on tasks normally managed by the spinal cord or adapt to a framework that is foreign by nature of the types of action and feedback control available to it. The brain is highly adaptable, but (like the designer of a cellular transplant) the designer of a prosthetic will ideally provide an interface, connectivity and means of control that do not depart too radically from normal intact adult function. The prosthetic control system may be built as an adaptive controller and the neural controls the prosthetic interacts with certainly are also plastic and adaptive systems. This presents a range of issues in control design and engineering that must be solved and better understood. A significant set of issues in the design of the prosthetic revolve 
around the degrees of freedom problem and CNS modularity. Depending on the level of interaction of the prosthetic and its nature (e.g., a spinal-cord neural driven smart leg orthosis for an amputee or SCI victim), the degrees of freedom problem can be very severe. The goal of smart prostheses that do not "dumb down" the subtlety and elegance of biological motor control is to face this issue.

\section{Prosthetic targets, technologies and their uses}

Both acquisitions of signals and stimulation of the nervous system form important components of current prostheses. The process of implanting and stabilizing electrodes can support either application, but the detailed fine design of the electrodes will differ based on application. We hope the reader will keep this in mind throughout the survey presented in this section. Both invasive $^{23}$ and noninvasive ${ }^{24}$ brain machine interface systems are extensively covered elsewhere. These may form a major source of user signals in future spinal prostheses. However, a range of interactions in the segmental motor system are also needed and can be achieved in a variety of ways. These are all areas of development and improvement. Our treatment here includes both clinical and animal model device developments.

\section{Peripheral prostheses}

The first class of prosthetic systems interact in the peripheral segmental nervous system (e.g., the Freehand system previously discussed). Extensive reviews are provided by Navarro and colleagues, ${ }^{25}$ Bhadra and colleagues, ${ }^{26}$ Peckham and Nutson, ${ }^{27}$ and Kilgore and colleagues. ${ }^{28}$ In general, there is a trade-off between invasiveness and the dual goals of selectivity and stability in peripheral interface designs. In degree of invasiveness, these range from surface electrodes through implanted sieve electrodes in which a peripheral nerve is cut and grown through a grid. For any of these motor prostheses, anterior horn cells must be intact.

Surface systems. Both muscle and peripheral nerve stimulation and (EMG) recording can be achieved from the skin surface. ${ }^{25,26}$

Transcutaneous muscle or nerve stimulators as output. These are routinely used in rehabilitation applications, and are often used in preliminary research or initial testing for fitting of prostheses. Their use may cause some pain or unwanted skin sensation and cosmesis is poor. Nonetheless, many candidates for prostheses avoid the invasive systems in favor of these where feasible, and their use is easily reversible. ${ }^{25,26}$

Surface EMG as a signal source. For myoelectric control, the noninvasive nature of surface EMG is significantly outweighed by the difficulties of calibration, variability and potential fragility of the recordings. However, surface EMG is nonetheless used as a control input in prostheses for foot drop and arm prosthesis. Multichannel high-density surface EMG augmented with modern signal processing approaches may considerably improve the information obtained. ${ }^{61}$

Percutaneous systems. Percutaneous systems can target deep muscles not accessible to transcutaneous systems, and they have minimal stimulation-associated pain. They can be used to assess patient suitability for implanted prosthetic systems, and they can be quite robust. ${ }^{27}$

Indwelling Systems. Chronic applications ultimately require indwelling systems. A range of technologies can now support these, with more technologies on the horizon.

Epimysial electrodes. Epimysial electrodes allow chronic access to deep muscles and have been very successfully applied in the Freehand hand-grasp system ${ }^{27}$ and also for standing. ${ }^{62}$ However, these electrodes suffer the problem of nonphysiological motor unit recruitment and consequent fatigue.

Intramuscular electrodes. BIONs as intramuscular electrodes are reviewed in another contribution to this volume.

Epineural electrodes. These electrodes comprise recording or stimulating sites on a longitudinal strip of biocompatible material such as Dacron-reinforced silicone rubber that is gently sutured to the epineurium. ${ }^{25}$ Alternatively, cylindrical electrodes can be percutaneously placed. ${ }^{64}$

Circumneural or nerve cuff electrodes. ${ }^{27,65}$ Helical cuffs are readily implanted and present little stress on the nerve, but they have less selectivity than split cylindrical or spiral cuffs. Spiral cuffs are carefully fitted to the nerve (e.g., those used clinically for vagal nerve stimulation) and provide selectivity ${ }^{66}$ due to the insulating material, but they may generate nerve damage if they are not carefully fitted. ${ }^{67} \mathrm{~A}$ range of electrode placements with cuffs is now extremely well-characterized, with tripolar being preferred in practice. Potentially, new technologies and materials might further improve on the cuff design. ${ }^{22,68}$ Ideally, focused recruitment of small fascicles is needed in all peripheral nervous system stimulation applications.

Flat interface neural electrodes (FINE). Nerves are malleable and stretchable on a longer time scale ${ }^{69}$ and a novel design uses this observation ${ }^{70}$ to gradually invade a nerve or more recently, to flatten a nerve over time. The result is that fascicles become arranged in a ribbon cable like geometry rather than as a cylindrical cable as often is routinely observed. However, in fact, some nerves are physiologically flattened already in vivo. This approach makes focused fascicle stimulation more feasible, using a flat interface multi-electrode cuff.

Intrafascicular (longitudinal penetrating) electrodes. An alternate strategy to surrounding and iso- 
lating bundles of nerve fascicles is to insert electrodes among them. This approach has been pioneered by Yoshida and Horsch. ${ }^{71}$ Applications have been limited as yet.

Prong/tine (transverse penetrating) electrodes.

Several approaches to multi-tine electrodes have been developed. Branner, Normann and colleagues ${ }^{72}$ have developed the Utah array comprising 100 electrodes in a regular $10 \times 10$ grid of tines customized for peripheral nerves. Such arrays, pneumatically inserted into the peripheral nervous system (e.g., into cat sciatic), provide long-term selective stimulation capabilities, but recording capacities have been, in contrast, significantly shortlived (i.e., only for a few weeks). ${ }^{73}$

Sieve (cut nerve) electrodes. Edell ${ }^{74}$ and Stieglitz and colleagues ${ }^{75}$ have independently explored a range of sieve electrodes in which a nerve is severed and grown through a sieve of recording sites. This approach likely has its main applications in amputees or perhaps in providing substituted peripheral feedback routes above a spinal transection that can pass through and be processed by all the normal somatosensory way stations.

Photic stimulators. Light has been tested as a peripheral nerve stimulation device. ${ }^{76,77}$ The packaging and clinical use remain unclear, but this method under development requires no special transfection or chromophore and channel insertions. Specificity of fascicle targeting within a nerve with such stimulation remains to be developed.

\section{DORSAL ROOT GANGLIA STIMULATION AND RECORDING}

Dorsal root ganglia have been recorded using multitine Utah arrays and "hatpin" electrodes (originally named for their sharp probe and electrode, protruding from a blunt, rounded base that sits on top of the pia, and its similarity to a 19th or early 20th century "lady's hatpin"), but chronic stability issues in the recording applications still need to be fully addressed.

\section{SURFACE SPINAL CORD AND ROOTS}

Targeting spinal cord for stimulation and recording of signals around or below a spinal transection may be feasible. However, the intraspinal experimental systems used in animal models remain fraught with difficulties under chronic conditions due to the motions and mechanical environment in the spinal canal. However, epidural stimulation is well established, and experimental designs to improve it or allow focused spinal electrode stimulation with flexible surface arrays are under way.

Epidural stimulation. Epidural paddle stimulators serve a range of purposes as discussed in other articles in this volume. They may be preferable to percutaneous epidural stimulators despite the increased invasivness. ${ }^{78}$ In animal models and in some human tests, epidural stimulators can activate pattern generators and may contribute to controlling extensor and antigravity tone.

Spinal surface systems. Flexible electrode systems suitable for wrapping over or around parts of the pia and adjacent to the spinal cord are under development in several laboratories. See Spence et al. ${ }^{79}$ for an unusual use.

\section{INTRASPINAL INTRAMEDULLARY DEVICES}

Intraspinal intramedullary electrodes are fraught with difficulty in chronic application. However, several designs have been used acutely or chronically. The McCreery laboratory has developed custom built tines to obtain the penetration needed for sacral bladder control in a cat model. ${ }^{80}$ The Utah design has been used acutely in the rat spinal cord by Yang and colleagues. ${ }^{81}$ The Utah design was used by Yang, working with Jack Judy and the Edgerton group. The depth of penetration of the Utah design and the extensive motion of cord during locomotion are issues being addressed. Microwires have been extensively tested, but with mixed results, in the cat spinal cord by Mushahwar and colleagues. ${ }^{82}$ In general, these have been used with a goal of deep placement among the motoneuron pools. The main difficulties, besides long-term stability, are precise targeting with microwire approaches. Michigan probes are flat silicon wafer single or multi-tine electrodes that are now commercially available to laboratory investigators. ${ }^{83}$ These are effective in the spinal cord in frogs and cats in acute conditions, but these are again difficult to use chronically.

Floating systems. "Floating electrode" implants are the "holy grail" of neuroprostheses. Ideally, dense recording sites are integrated into the tissue so they "float" or move freely with the implanted tissue as needed. ${ }^{84}$ Brain accelerations of several times gravity can occur in active behaviors, and the flexing and motion that occurs in the spinal cord is even more severe. One method to achieve a floating electrode assembly that is receiving considerable effort is the use of wireless telemetry and teleoperation with integrated on chip amplification and communication added to the embedded electrode system. Great strides are being made in this area. ${ }^{85}$ An alternate approach is to make the electrodes and wire leads capable of significantly more compliance than possible in current electrode designs. Working with materials and textiles experts, we (Giszter and Ko, unpublished results) have been exploring the use of braided and woven composites, which can be jammed or unjammed and altered in vivo as an electrode manufactured approach. For the spinal cord, in particular, this technology, which is now under United States and international patent review, may 
offer significant improvements over more conventional designs for floating electrode systems.

\section{FUTURE DIRECTIONS IN PROSTHESES FOR SCI}

Several approaches are in the experimental stages, but offer considerable near-term promise.

\section{Spinal stimulation for residual functions}

There are several strategies of central stimulation that have now been explored.

Epidural stimulation. Although spinal circuitry for organizing various types of movement exists in the spinal cord, these circuits are quiescent in mammals, unless strongly activated in some fashion. In the intact system, a cocktail of neuromodulators and descending control act to recruit and steer the circuitry as previously discussed. After adult spinalization, cats or rats require tail pinch or perineal stimulation to induce stepping through the pattern generators. In man, epidural stimulation can induce stepping. ${ }^{86}$ It has been explored in animal models as a means of activating lumbar pattern generator functions after spinal cord injuries. ${ }^{87-89}$ Implanting epidural stimulators above the lumbar and sacral segments, Edgerton and colleagues ${ }^{88}$ discovered that they were able to activate pattern generation and stepping at $\mathrm{L} 2$, and increase antigravity extensor forces at $\mathrm{S} 1$ in rats. The process of recovery and recruitment of bipedal treadmill stepping with epidural stimulation was paralleled by recovery of polysynaptic reflexes. ${ }^{87}$ Their data parallel data in the cat using the dorsal column, dorsal root and intraspinal microstimulation to induce stepping in cats. ${ }^{90,91}$ Epidural stimulation is a relatively coarse but effective means of engaging spinal circuitry. Alternative methods of potentially greater precision must penetrate and stimulate within the spinal cord.

Intraspinal stimulation. The potential advantages of electrical stimulation within the spinal cord are several-fold. First, stimulation of interneuronal or afferent systems rather than motor nerves has the potential to recruit target motor pools in size order and finesse issues of recruitment order and fatigue that can limit therapeutic effect in peripheral stimulation. Second, to the extent that spinal circuitry is appropriately engaged by stimulation, the powerful integration of information built into biological motor control solutions (i.e., pattern-generating circuits, reflexes, primitives) may be used. Finally, greater precision of control may be possible by accessing all spinal circuitry at multiple levels, if the technology permits. Early efforts in basic science and animal models have shown that intraspinal stimulation can be used to recruit pattern generation, ${ }^{92}$ and modular elements or primitives, ${ }^{93-95}$ as well as motor pools with a more natural ordering. ${ }^{96}$ The stimulation seems to tap into and recruit a modularity that is also being established by other means in both animal ${ }^{97,98}$ and human research. ${ }^{99}$

The potential power of intraspinal microstimulation (ISMS) is limited by our incomplete understanding of spinal circuitry and topography of the immediately recruited target elements and the interactions among multiple stimulated sites. In spinalized frogs ${ }^{100}$ and chronically spinalized rats, ${ }^{94}$ motor pool recruitment by singlepoint ISMS seems to have a crude topography. This is also seen in cats. ${ }^{90,91,95,101}$

In several studies, ${ }^{93,94,95,100}$ individual motion elements were identified with the trains of stimulation used, which was restricted to grey matter. As a result of chronic transection and deafferentation in frogs and rats in these studies, we know part of the ISMS results come from propriospinal system activation, not simply afferents, although these are also likely involved when present. These studies focused on ipsilateral isometric force and EMG responses. Several classes of movement elements were found in Barthelemy and colleagues, ${ }^{90}$ who stimulated both white and grey matter, used prolonged trains, and recorded kinematic and EMG responses as follows: ipsilateral nonlocomotor motions, bilateral and contralateral nonlocomotor motions, contralateral locomotor motions and bilateral locomotion. Barthelemy and colleagues ${ }^{90}$ were especially interested in locomotion and detailed topography in their cats.

Barthelemy and colleagues ${ }^{90,91}$ found a motor response topography using single-site train stimulation in their cats. However, it is also clear that the topography of ISMS results may be state and experience dependent. They found that trained chronically spinalized cats differed in some regards from untrained chronic spinalized cats. However, these differences more or less disappeared after clonidine administration. Similarly, Boyce, ${ }^{102}$ working with Lemay, found that after different therapies using combinations of cellular transplants and training in chronic spinalized cats, the proportions of representations of different response types altered. Boyce, ${ }^{102}$ working with Lemay, did not explore clonidine effects on "normalizing" maps. In contrast to the chronic spinalized cat studies of these groups, more rapid examinations of responses in the period of spinal shock, in spinally intact decerebrate, and in intact cats the results have emphasized the differences in topography. ${ }^{103}$ Taken together, from these studies it seems clear that the descending systems in the intact mammal normally modulate the spinal state and the susceptibility and effects of microstimulation can vary. However, a base topography, related in some way to the underlying circuits, may exist and may be available for ISMS after SCI. Modern management of SCI happily has the result that most injuries are now clinically incomplete. For this reason, the effects of descending systems and the spinal state on single and multiple electrode ISMS is a crucial 


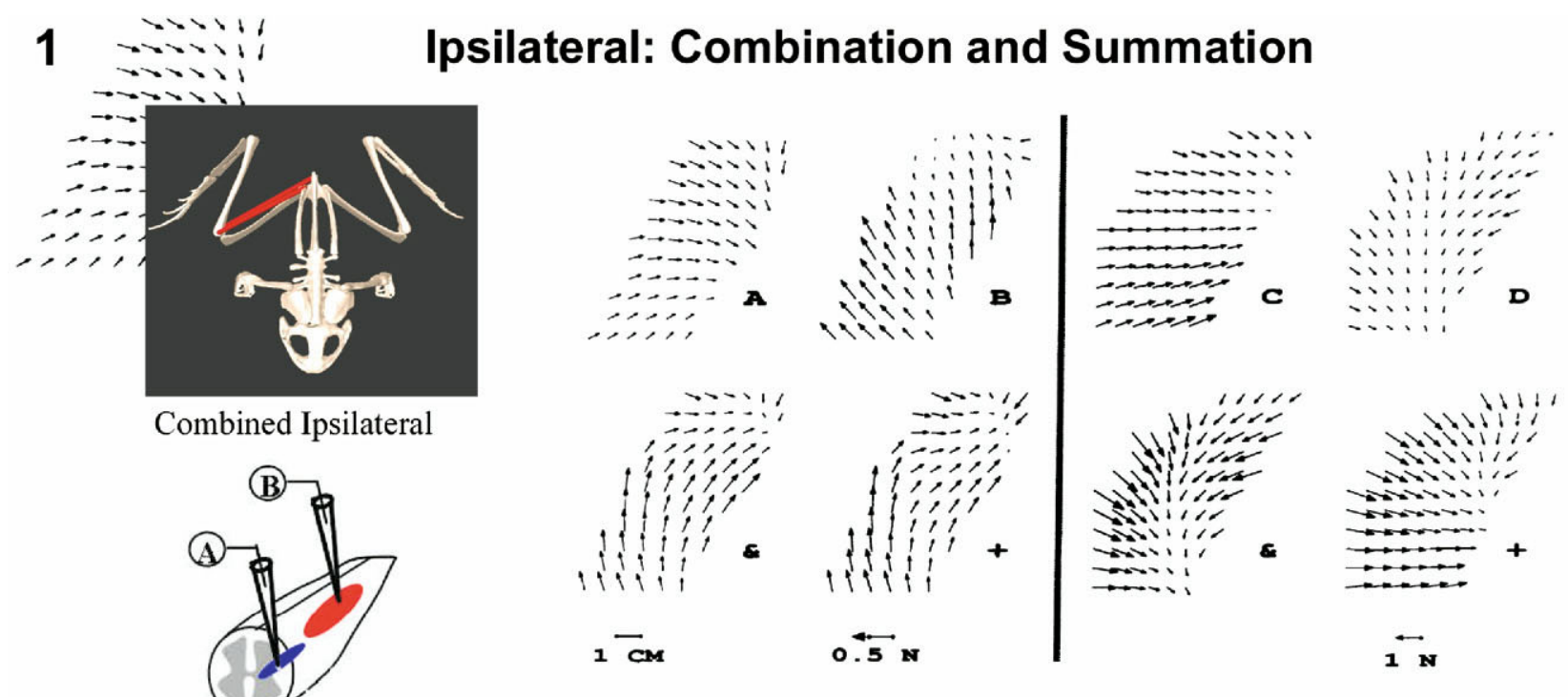

\section{$2 \quad$ Contralateral : Summation and non-linear interactions}
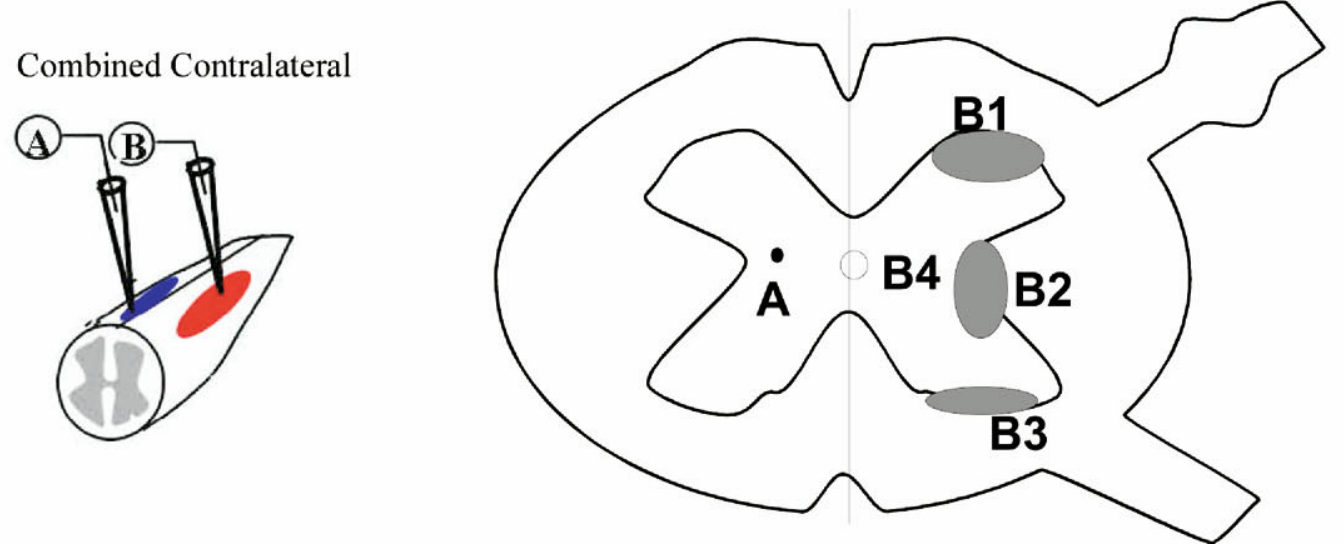

FIG 3. Intraspinal microstimulation below a lesion, possibilities and pitfalls. Panel 1: from Mussa-Ivaldi FA, Giszter SF, Bizzi E. Linear combinations of primitives in vertebrate motor control. Proc Natl Acad Sci USA 1994;91:7534-7538 (reproduced with permission). Results of co-stimulation of the lumbar gray matter in a frog. Fields are oriented to the frog as shown on far left. The hip of the frog is to the lower right in each field. Two results are found: (A) vector summation ( $\sim 85 \%$ of cases) and (B) winner-take-all suppression ( $\sim 15 \%$ of cases). Left: fields in relation to a frog. Middle: an example of vector summation. Upper left: fields obtained by separate stimulation of two sites, A and B. Lower left: co-stimulation fields (\&) and summation field (+). This was by far ( $87.8 \%$ of cases) the most common outcome. The quality of fitis determined as the inner product derived Cosine. Cos(A/B) close to 1.0 indicate near identity of two tested fields, a and b. In this panel Cos $(\& /+)=0.967$. Right: an example in which the co-stimulation (\&) did not correspond to the sum (+) of the two fields (C and D), but instead to a "winner-take-all" case ( $D$ is the winner). Cos $(D / \&)=0.949$; Cos $(+/ \&)=0.770$. Panel 2: redrawn from Giszter, Grill, Lemay, Mushahwar and Prochazka as a summary (with permission). Contralateral electrodes in combination in frogs may cause nonlinear effects as well as vector summation and winner-take-all suppression. Electrode $A$ was fixed in one hemicord, whereas B tracked through the other hemicord cord. A summary spinal cross section is shown. A, B and A+B were tested. If the combined stimulation $A+B$ had a significant force component out of the plane defined by $A$ and $B$, then the combined effect was not a summation, which would lie in the plane, but instead was highly nonlinear. Large regions of cord (shaded areas, B1, B2, B3) interacted nonlinearly, especially upper dorsal and lateral intermediate and deep ventral horn regions; although vector summation held across much of the frog spinal cord (B4).

issue. Multiple ISMS represents a richer but more complex means of controlling motor output in future therapeutic devices. ISMS will probably need to be tailored to the individual patient, depending on the degree of residual descending control.

Very promisingly, in single and dual stimulation in both frogs, ${ }^{104,105}$ rats $^{94}$ and cats, ${ }^{95}$ interactions among two sites in a hemicord were simple. Either a proportional vector summation or a winner-take-all interaction occurred among sites (FIG. 3). These interactions are consistent with what we understand of reflex and pattern generator construction of movement, where blends and switches occur $^{97,98,106}$ depending on the motor pattern, kinematic and kinetic context. The ISMS combination rules among stimuli applied simultaneously or in rapid succession at different stimulating electrode sites provide a basis or vocabulary for constructing novel movements and extending and adapting a basic reflex repertoire. 
There are several important gaps in our current knowledge of the results of such combinations. These gaps must be filled before ISMS can be used with confidence in therapeutic devices in clinical settings.

In decerebrate but nonspinal cats, vector summation was never observed. ${ }^{95}$ In both the chronically spinalized cats of Barthelemy and colleagues' 90 study, in which long stimulation trains were used, and in the chronic spinalized cats of Boyce's study, ${ }^{102}$ working with Lemay, in which shorter trains were used, dual site microstimulation was not examined. Thus, we do not know how the different sites interact in chronic spinalized cats, and we only have the Lemay and colleagues ${ }^{105}$ studies in decerebrate cats ${ }^{95}$ as a signpost.

In frogs, the interactions ipsilaterally are well characterized. Giszter and colleagues ${ }^{107}$ reported briefly on dual stimulation applied bilaterally. Bilateral interactions in spinal frogs included vector summation, winner-takeall suppression and a new class of responses in which nonlinear effects occurred. These nonlinear interactions indicate changes at one or both of the simultaneously stimulated sites and additional bilateral issues in ISMS. Understanding bilateral combination rules and multiple site interactions within the ISMS topography remain crucial as issues in ISMS device design. For example, multisite intraspinal stimulation for locomotion is unexplored, and the effects of its combination with other ISMS stimulation, which might be desirous for steering or anticipatory obstacle clearance, is unexplored. It is important to remember that any measured ISMS topography, although it derives from and relates to spinal organization, is simply a useful construct in device design, not a set of hypotheses about spinal cord or motor primitives. For example, basic science exploring the hypotheses of motor primitives has moved in other directions than ISMS. The needs of device construction, although informed by basic science, are unique and specific. From a device standpoint, ISMS effects remain to be examined in more detail. Technological limitations in chronic and acute electrode placement for ISMS continue to be limiting factors in this device development and exploration.

Stimulation in the motor pools with ISMS instead of the interneuronal regions of spinal cord is an alternate device construction strategy pursued by Mushahwar and colleagues. ${ }^{82}$ This may be very useful for fractionated movements, bypassing the bulk of spinal circuitry. It has recently been established that the primary stimulation effect in the motor pools seems to be by way of primary afferent axon recruitement. ${ }^{108}$ Since this afferent stimulation can potentially cause antidromic activation of the afferents and the processes of their dorsal columns, this stimulation strategy may not recruit motor pools with the purity initially supposed. ${ }^{96}$ Dorsal root afferents may also not be the only recruited elements. Various propriospinal systems might also be engaged by motor pool
ISMS. Thus, this method is probably subject to many of the same concerns and caveats discussed for other ISMS strategies and reviewed by Aoyagi and colleagues. ${ }^{103}$ Recently, investigations have also begun on intraspinal stimulation in the primate cervical enlargement. ${ }^{109}$

Bridging strategies. In principle, bridging strategies attempt to provide a high bandwidth link between the nervous system and the prosthetic or robotic device so as to bypass the lesion site. This approach is analogous to regeneration efforts in that it relies heavily and significantly on the intrinsic plasticity of the spinal and voluntary systems to coadapt in an appropriate fashion so as to support function. There is strong data supporting extensive cortical plasticity in novel brain machine interface tasks and supporting roles for cortical plasticity and substitution in recovery of locomotion after neonatal SCI in rats. However, developmental critical periods are no longer readily available in adults without additional measures, and the injured adult may face what Minsky and Selfridge ${ }^{110}$ has called the "Mesa problem." Learning improvements usually occur incrementally by approaching competency at a task using performance measures. The Mesa problem occurs when there are major discontinuities in the performance measure, similar to the mesas of the United States Southwest. Even close to a mesa, there is little or no local gradient information useful to indicate its proximity. Discovering and learning strategies in this landscape can then become very hard. Such a problem may exist in the novel cortico-prosthetic, cortico-robotic or cortico-spinal organization faced after spinal injury. Discontinuities in task space (e.g., upright weight-supported balance) that are overcome in normal development and with extensive childhood practice, may be next to impossible after a bridge intervention, unless special training regimes are instituted. Thus, for a thoracic injury prosthetic bridge, a combination of weightsupported robot training and augmentation of plasticity might be needed to integrate the bridge and learn its use in a functional manner. To date, this has not been welldemonstrated in animal models, which should clearly precede any clinical work.

Use of biomimetic robotics and neuromimetic computing in bridging or device interaction is becoming a viable possibility. Dobkin ${ }^{30}$ and others have suggested these may be a way station to full bridging and restoration. Rehabilitation training or augmentation of residual function with exoskeletal frames and/or biomimetic robotics and neuromimetic computational strategies ${ }^{111}$ may promote and expand functional capabilities. These are active areas of investigation with many questions remaining. In particular, the best ways to promote eventual independence of training or augmenting aids, rather than reliance, is an issue of great importance, in which the goal is that the therapeutic devices and robotic aids are only temporary. 

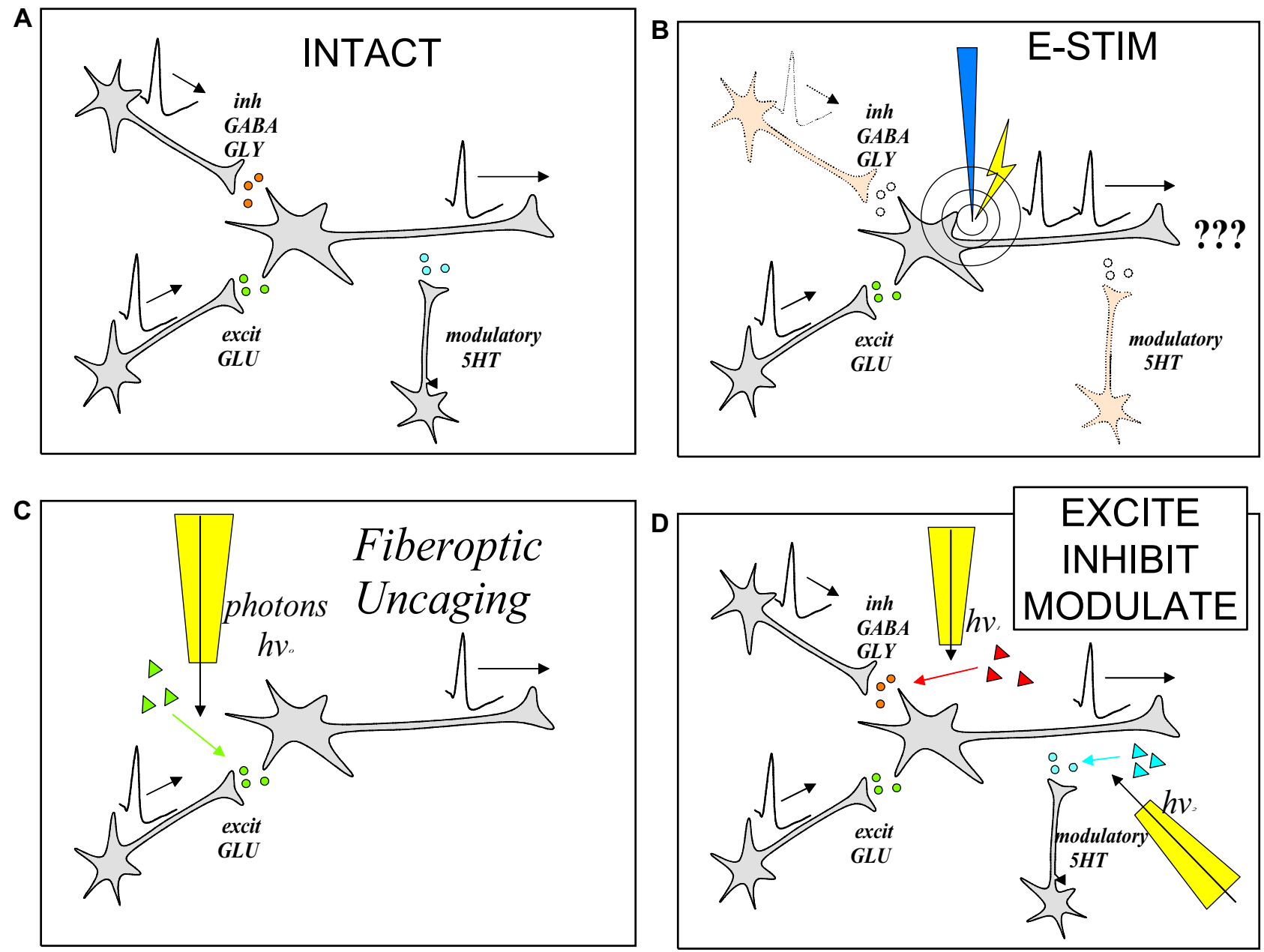

FIG 4. Future devices using optical methods. A: the intact CNS uses inhibitory, excitatory and modulatory synapses in specific temporal and spatial patterns. B: after spinal cord injuries, many modulatory and inhibitory pathways are lost. Stimulation electrically excite the axon hillock or axon and bypasses somatic integration and residual circuits. C: fiber optic uncaging or photo-activated channels offer(s) the possibility of controlling synaptic inputs. These are limited by light delivery methods and energetics, chemical caged transmitter stability, effective yield and tissue reuptake of transmitter and response to caged byproducts. D: the promise of caged compounds and wavelength specificity is the possibility of rapid spatially selective controls of inhibitory, excitatory and modulatory interactions in CNS targets. $5 \mathrm{HT}=5$ hydroxy-tryptamine (serotonin); E-STIM = electrical stimulation of axon; excit = excitatory; inh = inhibitory; GABA = gamma amino butyric acid; GLY = glycine; hv = uncaging photons.

\section{Rehabilitation approaches and future prostheses}

A range of robotic devices now exist for rehabilitation and training in the clinic (e.g., MIT Manus for the upper limb, ${ }^{112}$ Lokomat [Hocoma Co., Zurich, Switzerland] ${ }^{113}$ ). Some of these and other various active ortho$\operatorname{ses}^{54,114,115}$ might be adapted to prostheses use in the future. Presently, most of the focus is on the motions of limbs. However, trunk control and motion is a crucial component of intact movement planning and execution. Trunk prostheses or rehabilitation have only recently been tested. ${ }^{116,117}$

\section{Longer term needs for new device technologies}

Electrical stimulation, even microstimulation, is a crude instrument. The first structures recruited are fibers before cells. Recruitment order may differ strongly from that in neural activity-based processes. There are several newer means of control of neural tissues under investigation with their own advantages and problems. Photic stimulation of peripheral nerves has been demonstrated to be possible ${ }^{76,77}$ Photolytic uncaging of neurotransmitters ${ }^{118}$ from the covalently bonded chromophore moieties that render them biologically inert is now possible at wavelengths that are of little risk. Uncaging is a tradeoff of energetics of light delivered, speed of uptake of uncaged neurotransmitter by neural and glial scavenging systems, diffusion delivery and excitotoxic or other effects at extrasynaptic receptors (FIG. 4). The issues are complex and may be tissue specific. Long-term effects of the uncaged moieties must be understood and the route to FDA approval of both chemical and device components is likely to be long. An alternative approach is to insert photophores in target neurons: channels that can be light 
activated. ${ }^{119,120}$ Insertion can be achieved by a viral transfection with its attendant problems. Light-guide implantation, light couplings and their designs in photic systems present problems and challenges similar to wire implantation in electrical stimulation.

In electrical stimulation or photic stimulation, the issue is the probe or electrode constructions of high density, high compliance and high stability channels for light or electrical energy that can be accurately and precisely placed in the peripheral nervous system or CNS, despite the various tissue barriers and compliance variations, and chronic motions of tissues encountered. A range of new materials, fabrication approaches and nano-structured and micro-structured materials are being explored to attack this issue.

\section{CONCLUSIONS}

Prostheses for SCI in current use are likely to improve significantly over the next decade. Peripheral neural stimulation technologies are well established and understood, and peripheral recording is beginning to benefit from the multi-electrode approaches under development. Invasive and noninvasive brain machine interfaces as control signal channels are becoming available. Intraspinal stimulation is a potentially powerful approach, but requires significant technical advances and better understanding for clinical applications. ${ }^{121}$ The interaction of plasticity, both above and below the lesion, with devices, training and rehabilitation are of major therapeutic importance and focus for the future. Various technical improvements are on the horizon on several fronts, and various new avenues are being explored in stimulation and recording that will expand the therapeutic options.

Acknowledgments: The content of this article was strongly influenced by discussions at the National Institutes of Health Neural Interfaces Workshops and Neural Control of Movement Society Meetings. I am grateful to colleagues at Drexel University College of Medicine for suggestions, and Michel Lemay and Corey Hart, in particular, for discussion and reading of the manuscript, and to the volume editors and reviewers for their comments, especially Jason Schwalb. Supported by National Institutes of Health grant no. NS40412 and National Institutes of Health grant no. NS44564 and a Pennsylvania Tobacco Grid award.

\section{REFERENCES}

1. Schwab JM, Brechtel K, Mueller C-A, et al. Experimental strategies to promote spinal-cord regeneration- an integrative perspective. Prog Neurobiol 2006;78:91-116.

2. Pfister BJ, Huang JH, Kameswaran N, et al. Neural engineering to produce in vitro nerve constructs and neurointerface. Neurosurgery 2007;60:137-141.

3. Wolpaw JR. The education and re-education of the spinal cord. Prog Brain Res 2006;157:261-280.

4. Chapin JK, Moxon KA Neural prostheses for restoration of sensory and motor function. Boca Raton: CRC Press, 2000.
5. Leuthardt EC, Schalk G, Moran D, et al. The emerging world of motor neuroprosthetics: a neurosurgical perspective. Neurosurgery 2006;59:1-14.

6. de Leon RD, Kubasak MD, Phelps PE, et al. Using robotics to teach the spinal cord to walk. Brain Res Rev 2002;40:267-273.

7. Kilgore KL, Scherer M, Bobblitt R, et al. Neuroprosthesis consumers' forum: consumer priorities for research directions.J Rehabil Res Dev 2001;38:655-660.

8. Caggiano AO, Zimber MP, Ganguly A, et al. Chondroitinase $\mathrm{ABCI}$ improves locomotion and bladder function following contusion injury of the rat spinal cord. J Neurotrauma 2005;22:226239.

9. Houle JD, Tom VJ, Mayes D, et al. Combining an autologous peripheral nervous system "bridge" and matrix modification by chondroitinase allows robust, functional regeneration beyond a hemisection lesion of the adult rat spinal cord. J Neurosci 2006; 26:7405-7415.

10. Stirling RV, Dunlop SA, Beazley LD. Electrophysiological evidence for transient topographic organization of retinotectal projections during optic nerve regeneration in the lizard, Ctenophorus ornatus. Vis Neurosci 1999;16:681-693.

11. Dunlop SA, Stirling RV, Rodger J, et al. Failure to form a stable topographic map during optic nerve regeneration: abnormal activity-dependent mechanisms. Exp Neurol 2003;184:805-815.

12. Campos L, Ambron RT, Martin JH. Bridge over troubled waters. Neuroreport 2004;15:2691-2694.

13. Fawcett JW, Curt A, Steeves JD, et al. Guidelines for the conduct of clinical trials for SCI as developed by the ICCP panel: spontaneous recovery after SCI and statistical power needed for therapeutic clinical trials. Spinal Cord 2007;45:190-205.

14. U.S. Food and Drug Administration. Is the product a medical device? Available at: http://www.fda.gov/cdrh/devadvice/312. html. Accessed October 31, 2007.

15. Saviola J. The FDA's role in medical device clinical studies of human subjects. J Neurol Eng 2005;2:S1-4.

16. Falowski S, Celii A, Sharan A. Spinal cord stimulation: an update. Neurotherapeutics 2008;5:86-99.

17. Belverud S, Mogilner A, Schulder M. Intrathecal pumps. Neurotherapeutics 2008;5:114-122.

18. Mayer RD, Howard FM. Sacral nerve stimulation: neuromodulation for voiding dysfunction and pain. Neurotherapeutics 2008; 5:107-113.

19. Murphy DB, McGuire G, Peng P. Treatment of autonomic hyperreflexia in a quadriplegic patient by epidural anesthesia in the postoperative period. Anesth Analg 1999;89:148-149.

20. Yanagiya Y, Sato T, Kawada T, et al. Bionic epidural stimulation restores arterial pressure regulation during orthostasis. J Appl Physiol 2004;97:984-990.

21. Patil PG, Turner DA. The development of brain-machine interface neuroprosthetic devices. Neurotherapeutics 2008;5:137146.

22. Smart Prostheses: exploring assistive devices for the body and mind. Task Group Summaries. National Academies Keck Futures Initiative. The National Academies Press. 2007.

23. Donoghue JP, Nurmikko A, Black M, et al. Assistive technology and robotic control using motor cortex ensemble-based neural interface systems in humans with tetraplegia. J Physiol 2007;579: 603-611.

24. Lauer RT, Peckham PH, Kilgore KL. EEG-based control of a hand grasp neuroprosthesis. Neuroreport 1999;10:1767-1771.

25. Navarro X, Krueger TB, Lago N, et al. A critical review of interfaces with the peripheral nervous system for the control of neuroprostheses and hybrid bionic systems. J Peripher Nerv Syst 2005;10:229-258.

26. Bhadra N, Kilgore KL, Peckham PH. Implanted stimulators for restoration of function in SCI. Med Eng Phys 2001;23:19-28.

27. Peckham PH, Knutson JS. Functional electrical stimulation for neuromuscular applications. Annu Rev Biomed Eng 2005;7:327360.

28. Kilgore KL, Peckham PH, Keith MW. Advances in upper extremity functional restoration employing neuroprostheses. Ch 2 in neural prostheses for restoration of sensory and motor function. Chapin JK, and Moxon KA, ed. Boca Raton: CRC Press. 2000. 
29. Barbeau H, Ladouceur M, Mirbagheri MM, et al. The effect of locomotor training combined with functional electrical stimulation in chronic spinal cord injured subjects: walking and reflex studies. Brain Res Brain Res Rev 2002;40:274-291.

30. Dobkin B. The clinical science of neurologic rehabilitation, 2nd ed. New York: Oxford University Press. 2003.

31. Creasey GH, Kilgore KL, Brown-Triolo DL, et al. Reduction of costs of disability using neuroprostheses. Assist Technol 2000; 12:67-75.

32. Jacobs PL, Nash MS. Modes, benefits, and risks of voluntary and electrically induced exercise in persons with SCI. J Spinal Cord Med 2001;24:10-18.

33. Bogie KM, Triolo RJ. Effects of regular use of neuromuscular electrical stimulation on tissue health. J Rehabil Res Dev 2003; 40:469-475.

34. Agarwal S, Triolo RJ, Kobetic R, et al. Long-term user perceptions of an implanted neuroprosthesis for exercise, standing, and transfers after SCI. J Rehabil Res Dev 2003;40:241-252.

35. Petruska JC, Ichiyama RM, Jindrich DL, et al. Changes in motoneuron properties and synaptic inputs related to step training after spinal cord transection in rats. J Neurosci 2007;27:44604471.

36. Dupont-Versteegden EE, Houle JD, Gurley CM, et al. Early changes in muscle fiber size and gene expression in response to spinal cord transection and exercise. Am J Physiol 1998;275: C1124-C1133.

37. Ghiani CA, Ying Z, Vellis J, et al. Exercise decreases myelinassociated glycoprotein expression in the spinal cord and positively modulates neuronal growth. Glia 2007;55:966-975.

38. Vaynman S, Gomez-Pinilla F. Revenge of the "sit": how lifestyle impacts neuronal and cognitive health through molecular systems that interface energy metabolism with neuronal plasticity. J Neurosci Res 2006;84:699-715.

39. Wolpert DM, Ghahramani Z, Flanagan JR. Perspectives and problems in motor learning. Trends Cogn Sci 2001;5:487-494.

40. Giszter S, Patil V, Hart C. Primitives, premotor drives, and pattern generation: a combined computational and neuroethological perspective. Prog Brain Res 2007;165:323-46.

41. Davis DW, Thelen E, Keck J. Treadmill stepping in infants born prematurely. Early Hum Dev 1994;39:211-223.

42. Cappellini G, Ivanenko YP, Poppele RE, et al. Motor patterns in human walking and running. J Neurophysiol 2006;95: 3426-3437.

43. Grillner S. Biological pattern generation: the cellular and computational logic of networks in motion. Neuron 2006;52:751-766.

44. Kargo WJ, Giszter SF. Rapid correction of aimed movements by summation of force-field primitives. J Neurosci 2000;20:409426.

45. Giszter SF, Kargo WJ, Davies M, et al. Fetal transplants rescue axial muscle representations in M1 cortex of neonatally transected rats that develop weight support. J Neurophysiol 1998; 80:3021-3030.

46. Miya D, Giszter S, Mori F, et al. Fetal transplants alter the development of function after spinal cord transection in newborn rats. J Neurosci 1997;17:4856-4872.

47. Martin JH. The corticospinal system: from development to motor control. Neuroscientist 2005;11:161-173.

48. Bouyer LJ, Rossignol S. Contribution of cutaneous inputs from the hindpaw to the control of locomotion. II. Spinal cats. J Neurophysiol 2003;90:3640-3653.

49. Prinz AA. Insights from models of rhythmic motor systems. Curr Opin Neurobiol 2006;16:615-620.

50. Prinz AA, Bucher D, Marder E. Similar network activity from disparate circuit parameters. Nat Neurosci 2004;7:1345-1352.

51. Rossignol S, Brustein E, Bouyer L, et al. Adaptive changes of locomotion after central and peripheral lesions. Can J Physiol Pharmacol 2004;82:617-627.

52. Bouyer LJ, Whelan PJ, Pearson KG, et al. Adaptive locomotor plasticity in chronic spinal cats after ankle extensors neurectomy. J Neurosci 2001;21:3531-3541.

53. de Leon RD, Acosta CN. Effect of robotic-assisted treadmill training and chronic quipazine treatment on hindlimb stepping in spinally transected rats. J Neurotrauma 2006;23:1147-1163.
54. Reinkensmeyer DJ, Aoyagi D, Emken JL, et al. Tools for understanding and optimizing robotic gait training. J Rehabil Res Dev 2006;43:657-670.

55. Kirsch R. Development of a neuroprosthesis for restoring arm and hand function via functional electrical stimulation following high cervical SCI. Conf Proc IEEE Eng Med Biol Soc 2005;4:41424144.

56. Pearson KG. Generating the walking gait: role of sensory feedback. Prog Brain Res 2004;143:123-129.

57. Liberson WT, Holmquest HJ, Scot D, et al. Functional electrotherapy: stimulation of the peroneal nerve synchronized with the swing phase of the gait of hemiplegic patients. Arch Phys Med Rehabil 1961;42:101-105.

58. Graupe D. An overview of the state of the art of noninvasive FES for independent ambulation by thoracic level paraplegics. Neurol Res 2002;24:431-442.

59. Rybak IA, Stecina K, Shevtsova NA, et al. Modelling spinal circuitry involved in locomotor pattern generation: insights from the effects of afferent stimulation. J Physiol 2006;577:641-658.

60. Chen Y, Chen XY, Jakeman LB, et al. Operant conditioning of H-reflex can correct a locomotor abnormality after SCI in rats. J Neurosci 2006;26:12537-12543.

61. Zwarts MJ, Stegeman DF. Multichannel surface EMG: basic aspects and clinical utility. Muscle Nerve 2003;28:1-17.

62. Davis JA Jr, Triolo RJ, Uhlir J, et al. Preliminary performance of a surgically implanted neuroprosthesis for standing and transfers-where do we stand? J Rehabil Res Dev 2001;38:609-17.

63. Reference removed.

64. Slavin KV. Peripheral nerve stimulation for neuropathic pain. Neurotherapeutics 2008;5:100-106.

65. Hoffer JA, Kallesøe K. How to use nerve cuffs to stimulate, record or modulate neural activity. In: Neural prostheses for restoration of sensory and motor function. Chapin JK, Moxon KA, eds. Boca Raton: CRC Press, 2000:139-175.

66. Grill WM, Mortimer JT. Quantification of recruitment properties of multiple contact cuff electrodes. IEEE Trans Rehabil Eng 1996;4:49-62.

67. Grill WR, Mortimer JT. Stability of the input-output properties of chronically implanted multiple contact nerve cuff stimulating electrodes. IEEE Trans Rehabil Eng 1998;6:364-373.

68. Stieglitz T, Gross M. Flexible BIOMEMS with electrode arrangements on front and back side as key component in neural prostheses and biohybrid systems. Sensors Actuators B 2002;12:1-7.

69. Pfister BJ, Iwata A, Meaney DF, et al. Extreme stretch growth of integrated axons. J Neurosci 2004;24:7978-83.

70. Tyler DJ, Durand DM. Functionally selective peripheral nerve stimulation with a flat interface nerve electrode. IEEE Trans Neural Syst Rehabil Eng 2002;10:294-303.

71. Yoshida K, Horch K. Selective stimulation of peripheral nerve fibers using dual intrafascicular electrodes. IEEE Trans Biomed Eng 1993;40:492-494.

72. Branner A, Stein RB, Fernandez E, et al. Long-term stimulation and recording with a penetrating microelectrode array in cat sciatic Nerve IEEE Trans Biomed Eng 2004; 51:146-157.

73. Aoyagi Y, Stein RB, Branner A, et al. Capabilities of a penetrating microelectrode array for recording single units in dorsal root ganglia of the cat. J Neurosci Methods 2003;128:9-20.

74. Edell DJ. A peripheral nerve information transducer for amputees: long-term multichannel recordings from rabbit peripheral nerves. IEEE Trans Biomed Eng 1986;33:203-214.

75. Stieglitz T, Beutel H, Meyer J-U. A flexible, light-weightmultichannel sieve electrode with integrated cables for interfacing regenerating peripheral nerves. Sensors Actuators 1997;60:240243.

76. Wells J, Konrad P, Kao C, et al. Pulsed laser versus electrical energy for peripheral nerve stimulation. J Neurosci Methods 2007;163:326-37.

77. Wells J, Kao C, Konrad P, et al. Biophysical mechanisms of transient optical stimulation of peripheral nerve. Biophys J 2007; 93:2567-80.

78. North RB, Kidd DH, Petrucci L, et al. Spinal cord stimulation electrode design: a prospective, randomized, controlled trial com- 
paring percutaneous with laminectomy electrodes: part II-clinical outcomes. Neurosurgery 2005;57:990-996.

79. Spence AJ, Neeves KB, Murphy D, et al. Flexible multielectrodes can resolve multiple muscles in an insect appendage. J Neurosci Methods 2007;159:116-124.

80. McCreery D, Pikov V, Lossinsky A, et al. Arrays for chronic functional microstimulation of the lumbosacral spinal cord. IEEE Trans Neural Syst Rehabil Eng 2004;12:195-207.

81. Yang CT, Vaca L, Roy R, et al. Neural-ensemble activity of spinal cord L1/L2 during stepping in a decerebrate rat preparation. Proc. 2nd International IEEE /EMBS Conference on Neural Engineering; March 16-19, 2005; Arlington, Virginia.

82. Mushahwar VK, Collins DF, Prochazka A. Spinal cord microstimulation generates functional limb movements in chronically implanted cats. Exp Neurol 2000;163:422-429.

83. NeuroNexus Technologies. Available at: http://www.neuronexustech.com/. Accessed October 31, 2007.

84. Musallam S, Bak MJ, Troyk PR, et al. A floating metal microelectrode array for chronic implantation. J Neurosci Methods 2007;160:122-127.

85. Jackson A, Mavoori J, Fetz EE. Long-term motor cortex plasticity induced by an electronic neural implant. Nature 2006;444:56-60.

86. Dimitrijevic MR, Gerasimenko Y, Pinter MM. Evidence for a spinal central pattern generator in humans. Ann NY Acad Sci 1998;860:360-376.

87. Lavrov I, Gerasimenko YP, Ichiyama RM, et al. Plasticity of spinal cord reflexes after a complete transection in adult rats: relationship to stepping ability. J NeuroPhysiol 2006;96:16991710 .

88. Gerasimenko YP, Lavrov IA, Courtine G, et al. Spinal cord reflexes induced by epidural spinal cord stimulation in normal awake rats. J Neurosci Methods 2006;157:253-263.

89. Ichiyama RM, Gerasimenko YP, Zhong H, et al. Hindlimb stepping movements in complete spinal rats induced by epidural spinal cord stimulation. Neurosci Lett 2005;383:339-344.

90. Barthelemy D, Leblond H, Provencher J, et al. Nonlocomotor and locomotor hindlimb responses evoked by electrical microstimulation of the lumbar cord in spinalized cats. J NeuroPhysiol 2006;96:3273-3292.

91. Barthelemy D, Leblond H, Rossignol S. Characteristics and mechanisms of locomotion induced by intraspinal microstimulation and dorsal root stimulation in spinal cats. J NeuroPhysiol 2007;97:1986-2000.

92. Iwahara T, Atsuta Y, Garcia-Rill E, et al. Spinal cord stimulation induced locomotion in the adult cat. Brain Res Bull 1991;28:99105.

93. Giszter SF, Mussa-Ivaldi FA, Bizzi E. Convergent force fields organized in the frog's spinal cord. J Neurosci 1993;13:467-491.

94. Tresch MC, Bizzi E. Responses to spinal microstimulation in the chronically spinalized rat and their relationship to spinal systems activated by low threshold cutaneous stimulation. Exp Brain Res 1999;129:401-416.

95. Lemay MA, Grill WM. Modularity of motor output evoked by intraspinal microstimulation in cats. J NeuroPhysiol 2004;91: 502-514.

96. Mushahwar VK, Horch KW. Selective activation of muscle groups in the feline hindlimb through electrical microstimulation of the ventral lumbo-sacral spinal cord. IEEE Trans Rehabil Eng 2000;8:11-21.

97. Kargo WJ, Giszter SF. Rapid correction of aimed movements by summation of force-field primitives. J Neurosci 2000;20:409426.

98. Lafreniere-Roula M, McCrea DA. Deletions of rhythmic motoneuron activity during fictive locomotion and scratch provide clues to the organization of the mammalian central pattern generator. J NeuroPhysiol 2005;94:1120-1132.

99. Cappellini G, Ivanenko YP, Poppele RE, et al. Motor patterns in human walking and running. J NeuroPhysiol 2006;95: 3426-3437.

100. Bizzi E, Mussa-Ivaldi FA, Giszter S. Computations underlying the execution of movement: a biological perspective. Science 1991;253:287-291.
101. Tai C, Booth AM, Robinson CJ, et al. Multi-joint movement of the cat hindlimb evoked by microstimulation of the lumbosacral spinal cord. Exp Neurol 2003;183:620-627.

102. Boyce VS. Treadmill locomotor training and neurotrophic factors: their effect on locomotor recovery and spinal modularity in the chronic spinal cat [PhD thesis]. Philadelphia, Penn: Neuroscience Graduate Program, Drexel University College of Medicine; 2006.

103. Aoyagi Y, Mushahwar VK, Stein RB, et al. Movements elicited by electrical stimulation of muscles, nerves, intermediate spinal cord, and spinal roots in anesthetized and decerebrate cats. IEEE Trans Neural Syst Rehabil Eng 2004;12:1-11.

104. Mussa-Ivaldi FA, Giszter SF, Bizzi E. Linear combinations of primitives in vertebrate motor control. Proc Natl Acad Sci U S A 1994;91:7534-7538.

105. Lemay MA, Galagan JE, Hogan N, et al. Modulation and vectorial summation of the spinalized frog's hindlimb end-point force produced by intraspinal electrical stimulation of the cord. IEEE Trans Neural Syst Rehabil Eng 2001;9:12-23.

106. Stein PS, Camp AW, Robertson GA, et al. Blends of rostral and caudal scratch reflex motor patterns elicited by simultaneous stimulation of two sites in the spinal turtle. J Neurosci 1986;6: 2259-2266

107. Giszter SF, Grill WM, Lemay MA, et al. Intraspinal microstimulation: techniques, perspectives and prospects for FES. in Neural prostheses for restoration of Sensory and motor function. Moxon KA, Chapin, JK, eds. Boca Raton: CRC Press, 2000:101-138.

108. Gaunt RA, Prochazka A, Mushahwar VK, et al. Intraspinal microstimulation excites multisegmental sensory afferents at lower stimulus levels than local alpha-motoneuron responses. J NeuroPhysiol 2006;96:2995-3005.

109. Moritz CT, Lucas TH, Perlmutter SI, et al. Forelimb movements and muscle responses evoked by microstimulation of cervical spinal cord in sedated monkeys. J NeuroPhysiol 2007;97:110 120.

110. Minsky ML, Selfridge OG. Learning in random nets, in Fourth London Symposium on Information Theory. Cherry C, ed. NY: Academic Press, Inc, 1957.

111. Simoni MF, Cymbalyuk GS, Sorensen ME, et al. A multiconductance silicon neuron with biologically matched dynamics. IEEE Trans Biomed Eng 2004;51:342-354.

112. Fasoli SE, Krebs HI, Hogan N. Robotic technology and stroke rehabilitation: translating research into practice. Top Stroke Rehabil 2004;11:11-19.

113. Lunenburger L, Colombo G, Riener R, et al. Biofeedback in gait training with the robotic orthosis Lokomat. Conf Proc IEEE Eng Med Biol Soc 2004;7:4888-91.

114. Schmidt H, Werner C, Bernhardt R, et al. Gait rehabilitation machines based on programmable footplates. J Neuroengineering Rehabil 2007:4:2

115. Aaron RK, Herr HM, Ciombor DM, et al. Horizons in prosthesis development for the restoration of limb function. J Am Acad Orthop Surg 2006;14:S198-204.

116. Udoekwere UI, Ramakrishnan A, Mbi L, Giszter SF. Robot application of elastic fields to the pelvis of the spinal transected rat: a tool for detailed assessment and rehabilitation. Proceedings of the 28th Annual International Conference of the IEEE/EMBS 2006; November 2006, New York.

117. Ichinose WE, Reinkensmeyer DJ, Aoyagi D, et al. A robotic device for measuring and controlling pelvic motion during locomotor rehabilitation (man). Proc 25th Ann Intl Conf IEEE EMBS, 2003; Cancun, Mexico.

118. Haydon PG, Ellis-Davies GC. Ultrahigh-speed photochemical stimulation of neurons. Nat Methods 2005;2:811-812.

119. Arenkiel BR, Peca J, Davison IG, et al. In vivo light-induced activation of neural circuitry in transgenic mice expressing channelrhodopsin-2. Neuron 2007;54:205-218.

120. Deisseroth K, Feng G, Majewska AK, et al. Next-generation optical technologies for illuminating genetically targeted brain circuits. J Neurosci 2006;26:10380-10386.

121. Barbeau H, McCrea DA, O'Donovan MJ, et al. Tapping into spinal circuits to restore motor function. Brain Res Brain Res Rev 1999;30:27-51. 